\title{
Immunoelectron Microscopy Reveals Varied Surface Expression of Potential Vaccine Targets of Chlamydia trachomatis
}

\author{
Ru-ching Hsia ${ }^{1,2}$, Johanna Sotiris ${ }^{1}$ and Patrik Bavoil ${ }^{3}$
}

1. Electron Microscopy Core Imaging Facility, University of Maryland Baltimore, Baltimore, USA

2. Department of Neural \& Pain Sciences, University of Maryland School of Dentistry, Baltimore, USA

3. Department of Microbial Pathogenesis, University of Maryland School of Dentistry, Baltimore, USA

Chlamydia trachomatis, an obligate intracellular bacterium, is a leading cause of sexually transmitted infection in the world. It is estimated that $6.8 \%$ of sexually active young females aged 14-19 in the US may be infected with Chlamydia [1]. Previous studies have demonstrated that a nine-member $C$. trachomatis protein family, the polymorphic membrane proteins (Pmps), plays a critical role in pathogenesis and may be a potential vaccine target [2]. These proteins share specific structural motifs and are transported to the chlamydial surface via an autotransporter mechanism [2]. We report here on the differential subcellular location of two of these proteins, PmpD and PmpI, at different stages of chlamydial development using immuno electron microscopy.

C. trachomatis-infected HeLa cells were fixed at mid or late developmental stages during a $48 \mathrm{hr}$ infection cycle. Fixed monolayers were scraped off tissue culture vessels, enrobed in $2.5 \%$ low melting temperature agarose and trimmed into $\sim 1 \mathrm{~mm}^{3}$ pieces. Agarose pieces containing infected cells were cryo-protected with $30 \%$ sucrose, attached to a cryo-ultramicrotome specimen pin and flash frozen in liquid nitrogen, or dehydrated and embedded in unicryl resin using the progressive lowering temperature (PLT) method [3]. Immunolabeling was performed using either thawed cryo ultrathin sections following the Tokuyasu method [4], or unicryl ultrathin sections on grids.

At the mid developmental stage (18 hrs post infection), metabolically active chlamydial forms multiply within an inclusion as reticulate bodies (RBs). Immunogold particles labeling PmpI were distributed mostly on the RB membrane whereas the majority of gold particles labeling PmpD were located in the RB cytoplasm (Figure 1). Furthermore, a cluster of gold particles along one fraction of the RB membrane were occasionally observed both in PmpI and PmpD immunolabeling experiments. At the late developmental stage (45 hrs post infection), RBs transform into metabolically inert elementary bodies (EBs). The gold particles labeling PmpI were mostly found in the cytoplasm of EBs whereas those labeling PmpD were found on the outer surface of EB envelope (Figure 2). These results illustrate the differential spatial distribution of PmpI and PmpD at late developmental stages for C. trachomatis.

This study demonstrates the capability of immuno electron microscopy to reveal the differential subcellular location of PmpD and PmpI at different stages of chlamydial development. The presence of PmpD at the external surface of infectious chlamydial EBs suggest PmpD may be a better vaccine target. Our results indicate that PmpI and PmpD may perform different functions at different developmental stages and imply that the secretion of these two autotransported proteins may be regulated at different developmental stages. Last, the unique clustered distribution of PmpI and PmpD gold labeling strongly suggests the existence of a supramolecular structure at a specific location of the chlamydial surface. 


\section{References:}

[1] CDC Morbidity and Mortality Weekly Report 60 (2011), p. 370.

[2] D Crane et al, Proc Natl Acad Sci USA.103 (2006), p.1894.

[3] P Gounon, Methods Mol Biol.117 (1999), p.111.

[4] K Tokuyasu, Journal of Microscopy 143 (1986), p.139.

[5] The authors acknowledge funding from the NIH Shared Instrument grant, Grant Number 1S10RR26870-1.
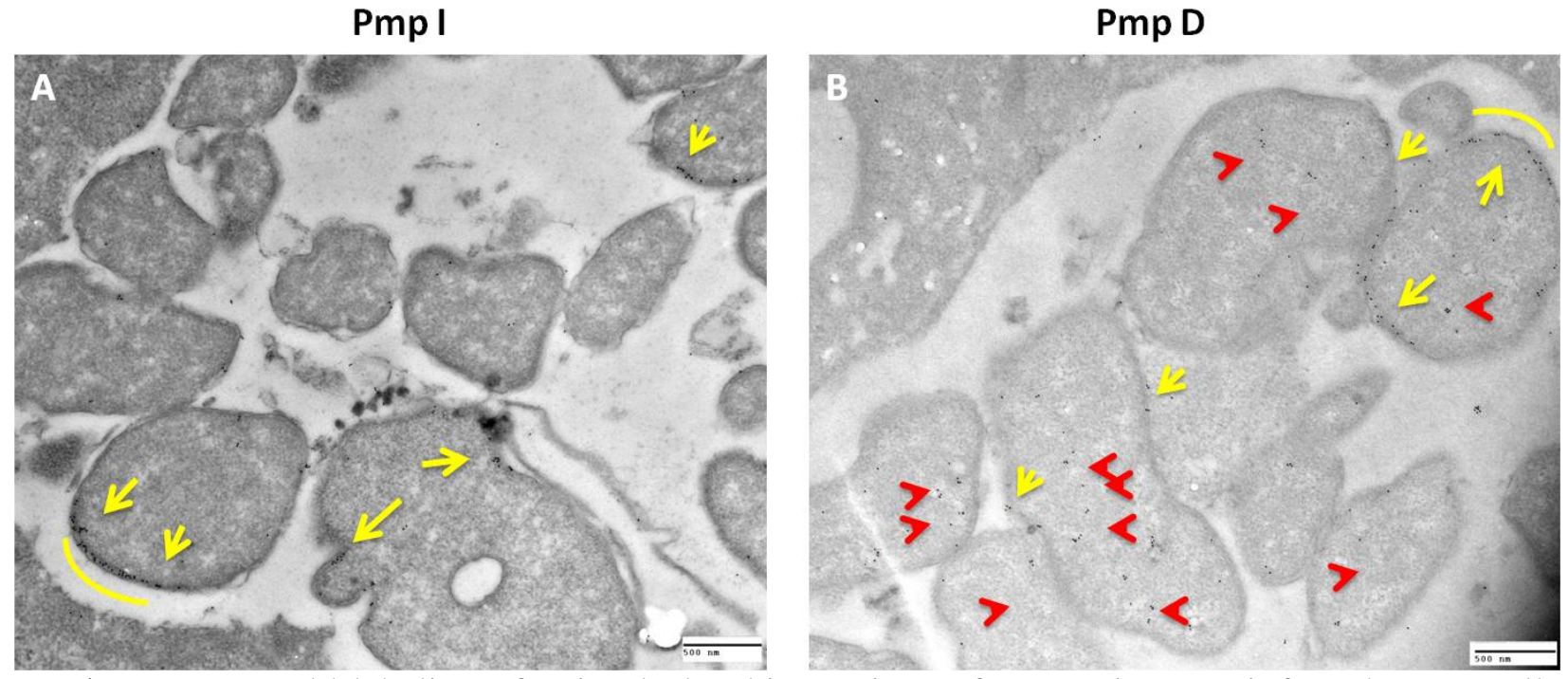

Figure 1. Immunogold labeling of unicryl ultrathin sections of C. trachomatis-infected HeLa cells 18 hrs post infection. Sections were labeled using primary antibody against PmpI (A) and PmpD (B) and a secondary antibody conjugated with $10 \mathrm{~nm}$ gold particles. Yellow arrows indicate gold particles on the chlamydial surface. Red arrowheads indicate gold particles in the cytoplasm. The yellow line indicates clustered gold particles along the RB surface. Bar, $500 \mathrm{~nm}$.

Pmp I

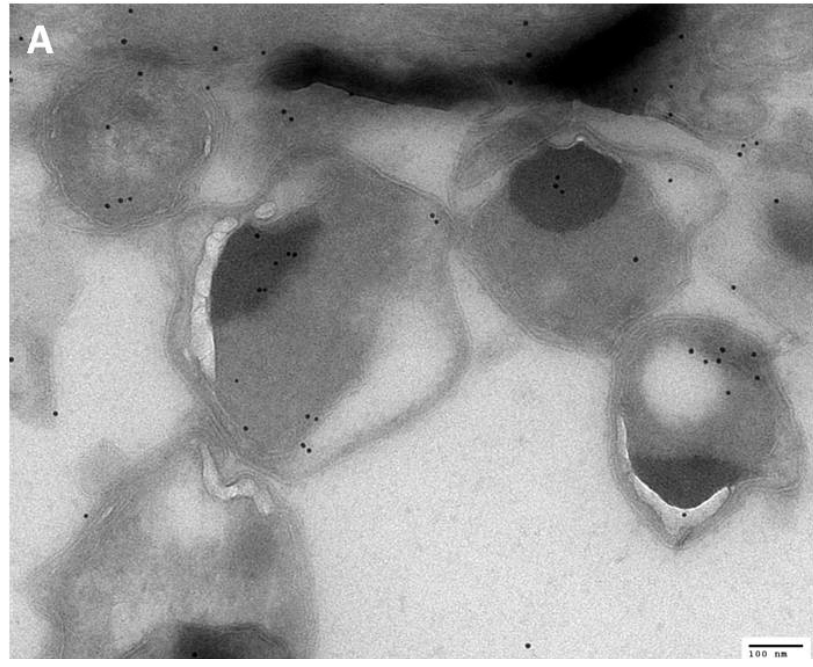

Pmp D

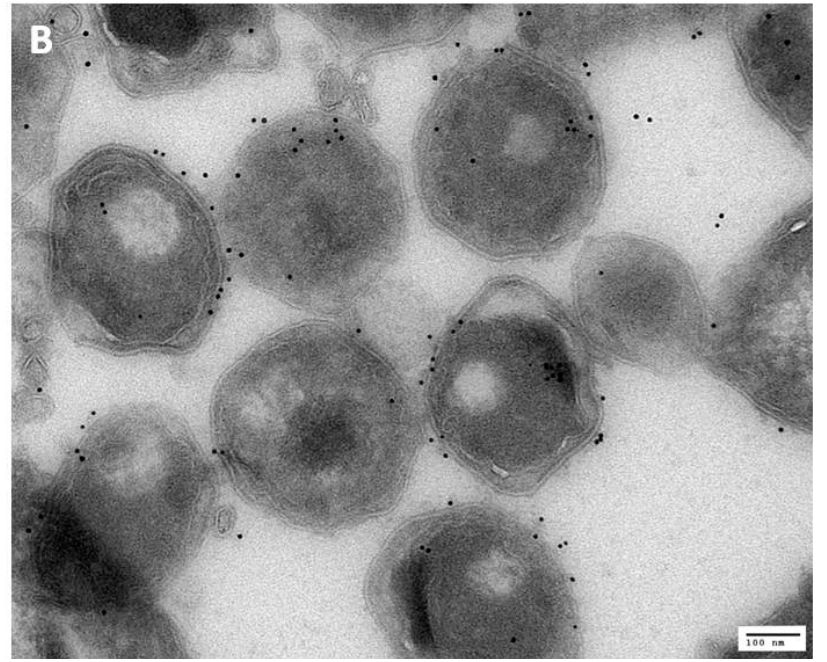

Figure 2. Immunogold labeling of thawed cryo-ultrathin sections of $C$. trachomatis-infected Hela cells at 44 hrs post infection. Sections were labeled using primary antibody against PmpI (A) and PmpD (B) and a secondary antibody conjugated with $10 \mathrm{~nm}$ gold particles. Bar, $100 \mathrm{~nm}$. 